\title{
Datos geográficos de los murciélagos (Chiroptera) en el Neotrópico
}

\author{
Elkin A. Noguera-Urbano \& Tania Escalante \\ Museo de Zoología “Alfonso L. Herrera”, Departamento de Biología Evolutiva, Facultad de Ciencias, Universidad \\ Nacional Autónoma de México. Apartado Postal 70-399, 04510 México, D. F., México; elkalexno@gmail.com, \\ tee@st.ib.unam.mx
}

Recibido 05-IV-2013. Corregido 08-IX-2013. Aceptado 03-X-2013.

\begin{abstract}
Geographic data for Neotropical bats (Chiroptera). The global effort to digitize biodiversity occurrence data from collections, museums and other institutions has stimulated the development of important tools to improve the knowledge and conservation of biodiversity. The Global Biodiversity Information Facility (GBIF) enables and opens access to biodiversity data of 321 million of records, from 379 host institutions. Neotropical bats are a highly diverse and specialized group, and the geographic information about them is increasing since few years ago, but there are a few reports about this topic. The aim of this study was to analyze the number of digital records in GBIF of Neotropical bats with distribution in 21 American countries, evaluating their nomenclatural and geographical consistence at scale of country. Moreover, we evaluated the gaps of information on $1^{\circ}$ latitude $\times 1^{\circ}$ longitude grids cells. There were over $1 / 2$ million records, but $58 \%$ of them have no latitude and longitude data; and 52\% full fit nomenclatural and geographic evaluation. We estimated that there are no records in $54 \%$ of the analyzed area; the principal gaps are in biodiversity hotspots like the Colombian and Brazilian Amazonia and Southern Venezuela. In conclusion, our study suggests that available data on GBIF have nomenclatural and geographic biases. GBIF data represent partially the bat species richness and the main gaps in information are in South America. Rev. Biol. Trop. 62 (1): 209-215. Epub 2014 March 01.
\end{abstract}

Key words: records, database, species number, richness, bats, Latin America.

La liberación de la información curatorial, taxonómica y geográfica de las bases de datos de las colecciones biológicas disponibles en internet (Escalante, 2005), ha abierto un nuevo enfoque para los análisis espaciales y la conservación de la biodiversidad (Gaubert, Papes \& Peterson, 2006). Una de las más grandes fuentes de consulta sobre información en biodiversidad es el portal de GBIF "Global Biodiversity Information Facility", el cual provee acceso libre a más de 383 millones de registros, a partir de datos contenidos en 9962 bases de datos y 456 organizaciones y museos alrededor del mundo (http://www.gbif.org/ visitado 25 de enero de 2013). Algunos beneficios del portal de GBIF son permitir el acceso a un alto número de registros y la repatriación de la información (Edwards, 2004). Sin embargo, pueden encontrarse diferentes problemas y errores en las bases de datos asociadas a GBIF (Meier \& Dikow, 2004; Chapman, 2005; Yesson et al., 2007; Costello et al., 2012), algunos de estos son la presencia de sinonimias, errores tipográficos y referencias geográficas incorrectamente relacionadas (Yesson et al., 2007; Costello et al., 2012). Así, una gran cantidad de datos disponibles en las bases de datos consultadas a través de la red, pueden no tener referencia geográfica o si la hay es imprecisa (Soberon \& Peterson, 2009); en muchos casos, los registros representan a algunos taxones y se concentran en unas pocas regiones (Yesson et al., 2007).

No obstante a los problemas que traen consigo el manejo de grandes bases de datos, en años recientes los avances tecnológicos y la liberación de registros a través de internet, han 
permitido el desarrollo de enfoques múltiples basados en su procesamiento. Algunas aplicaciones van desde análisis evolutivos (Kozak, Graham \& Wiens, 2008), función ecosistémica, cambio climático global, epidemiología, análisis taxonómicos, selección de áreas de conservación, hasta el diseño e implementación de políticas ambientales (Shaffer, Fisher \& Davidson, 1998; Ponder et al., 2001; Lane, 2007; Boitani et al., 2012).

Hacia el año 2010, la Red Latinoamericana y el Caribe para la Conservación de los Murciélagos formalizó una estrategia para la conservación de los murciélagos de América Latina y el Caribe, en el cual una de las metas es implementar un sistema de áreas de protección de los murciélagos de América Latina (AICOMs) y sus hábitats (RELCOM, 2010). Por lo tanto, se hace necesaria la evaluación de la información disponible en GBIF, considerando que podría ser una fuente potencial de datos para la conservación de los murciélagos en América Latina.

Aquí se propone abordar de una manera sistemática la revisión de información disponible en GBIF para los registros de murciélagos (Chiroptera) de 21 países neotropicales (Argentina, Belice, Bolivia, Brasil, Chile, Colombia, Costa Rica, Ecuador, El Salvador, Guatemala, Guyana, Guyana Francesa, Honduras, México, Nicaragua, Panamá, Paraguay, Perú, Suriname, Uruguay y Venezuela). Las interrogantes que se platean son: ¿Cómo se distribuyen los registros en los países latinoamericanos?, ¿cuáles y cuántas especies están representadas en dicha base de datos?, ¿cuáles países tienen la mayor cantidad de datos disponibles?, ¿cuáles son las áreas con la mayor o menor cantidad posible de información sobre murciélagos neotropicales disponible en GBIF?

Los registros facilitados por GBIF principalmente proveen información taxonómica y geográfica de los ejemplares que representan, por lo tanto se exploran los dos tipos de validez, errores y aciertos del tipo nomenclatural en cuanto al nombre científico y errores geográficos de relación o no relación a escala de país, empleando listas de especies y polígonos de los países para realizar las validaciones.

\section{MATERIALES Y MÉTODOS}

Captura de información: Se realizaron consultas en el portal de GBIF (http://www. gbif.org) para compilar la información sobre datos espaciales de murciélagos (Chiroptera) del Neotrópico, considerando como criterios de búsqueda principal el orden "Chiroptera" y el país (Fecha de acceso 20-21/07/2012). Se restringió la búsqueda a 21 países que hacen parte del Neotrópico continental americano, incluyendo a Argentina, Belice, Bolivia, Brasil, Chile, Colombia, Costa Rica, Ecuador, El Salvador, Guyana Francesa, Guatemala, Guyana, Honduras, México, Nicaragua, Panamá, Paraguay, Perú, Suriname, Uruguay y Venezuela. GBIF hace una revisión de la concordancia semántica de textos con listados preestablecidos (nombres científicos, nombres de países y localidades), de este proceso resultan columnas con nombres interpretados. Por lo tanto, para cada país se descargaron bases de datos que incluyeron los campos: Nombre científico interpretado (Scientific name [interpreted]), país interpretado (Country [interpreted]), latitud y longitud. Se cuantificó el número de registros georreferidos y sin georreferir y el número de variaciones en los nombres científicos para cada país y en todos los países.

Evaluación del nombre: Se realizó la evaluación considerando el campo "Nombre científico interpretado". Aunque algunos registros presentaron la categoría taxonómica de subespecie y otros en el mismo campo incluyeron el autor de la especie, todos fueron tratados como especies (género+epíteto específico). La evaluación fue realizada comparando los nombres científicos de los murciélagos descargados de GBIF, con una lista de referencia que incluyó las propuestas de Wilson \& Reeder (2005), Simmons (2005) y Gardner (2008). Además, se consideraron modificaciones realizadas por Solari et al. (2009) para Dermanura; Solari \& Baker (2006) y Zurc \& Velazco (2010) para 
Carollia; Velazco (2005), Velazco \& Gardner (2009) y Velazco, Gardner \& Patterson (2010) para Platyrrhinus, y Velazco \& Simmons (2011) para Vampyrodes. Se extrajeron y almacenaron en una nueva base de datos aquellos registros con nombres coincidentes con la lista de referencia. La nueva base de datos fue visualizada como mapa de puntos en ArcGIS ${ }^{\circledR} 9.3$ (ESRI, 2008). Se contabilizaron los nombres de las especies antes y después del proceso. El manejo de los datos se realizó en Microsoft Excel 2007 y herramientas del complemento de Excel "ddTraDa Básico" Versión libre 1.54 (de Diego, 2010).

Evaluación geográfica: La base de datos obtenida en la evaluación de nombre fue subdividida por países considerando como filtro el campo de GBIF "País interpretado", esto con el objetivo de obtener una aproximación de la correspondencia espacial de cada registro a escala de país. La validación se realizó con polígonos administrativos de cada país, obtenidos de la base de datos mundial sobre áreas administrativas (GADM 2.0 2011). Cada subconjunto de datos conformado por los registros de murciélagos (Chiroptera) en archivo shapefile fue superpuesto sobre el polígono del país correspondiente. Se almacenaron en una nueva base de datos aquellos registros coincidentes con los polígonos administrativos (Escalante, Espinosa \& Morrone, 2002; Chapman, 2005). El número de registros anterior y posterior a la evaluación geográfica fue representado en diagramas de frecuencias. Los análisis de superposición y proyección de mapas se realizaron en ArcGIS ${ }^{\circledR} 9.3$ (ESRI, 2008). Los nombres obtenidos fueron comparados con la riqueza de murciélagos en cada país y otros recursos geográficos disponibles como los mapas de la Unión Internacional para la Conservación de la Naturaleza (IUCN).

Análisis espacial de registros y especies: Se realizó un análisis exploratorio del número de especies y registros sobre una cuadrícula de $1^{\circ}$ de latitud $\times 1^{\circ}$ de longitud (Petersen, Meier \& Nykjaer, 2003; Yesson et al., 2007) para determinar áreas con concentración de registros y especies. Se consideraron únicamente los datos obtenidos luego de la validación de nombre y relación geográfica. La creación de la cuadrícula y el análisis de número de registros y especies sobre la cuadrícula, fueron realizados empleando las herramientas de DIVA-GIS Versión 7.5 (Hijmans et al., 2004) y ArcGIS 9.3® (ESRI, 2008). Se proyectaron los resultados como mapas (Crisp et al., 2001) reclasificados con intervalos de clases iguales. Se obtuvo un análisis de correlación de Spearman (Sokal \& Rohlf, 1995), para evaluar la relación entre número de especies y registros. La prueba estadística se realizó en el programa $\mathrm{R}$ versión 2.14.1 (2011), paquete Raster (Hijmans \& van Etten, 2012).

\section{RESULTADOS}

Captura de información: La búsqueda en GBIF resultó en 449371 registros de murciélagos con distribución en 21 países del Neotrópico (Anexo I. Contiene la lista de instituciones fuente). Del total de registros, el 58.5\% (262 889 registros) estuvieron georreferidos (Fig. 1), mientras que el 41.5\% (186 482 registros) no presentaron georreferencia. Se presentaron grandes diferencias con respecto al número de registros entre países (Fig. 2A; Cuadro 1). La mayoría de los registros se encontraron para cuatro países $(66 \%)$ : México que tuvo el mayor número de registros (166 391 registros totales/123 950 registros georreferidos), seguido por Colombia (48 362/14 899), Venezuela (43 915/37 832) y Panamá (38 270/9 412). Suriname tuvo el menor número de registros (1482 / 695), seguido por Chile (520/356) y Uruguay (514/138). Se obtuvieron 2976 nombres de especies en el área analizada, que incluyeron tanto nombres actualmente válidos, como con errores tipográficos, sinónimos y determinaciones hasta taxones supraespecíficos diferentes. El número total de nombres en cada país fue variable (Cuadro 1), en los registros de México estuvo el mayor número de variaciones de nombres con 1087 (Fig. 2B), seguido por Perú (368) y Colombia (323). Los registros 

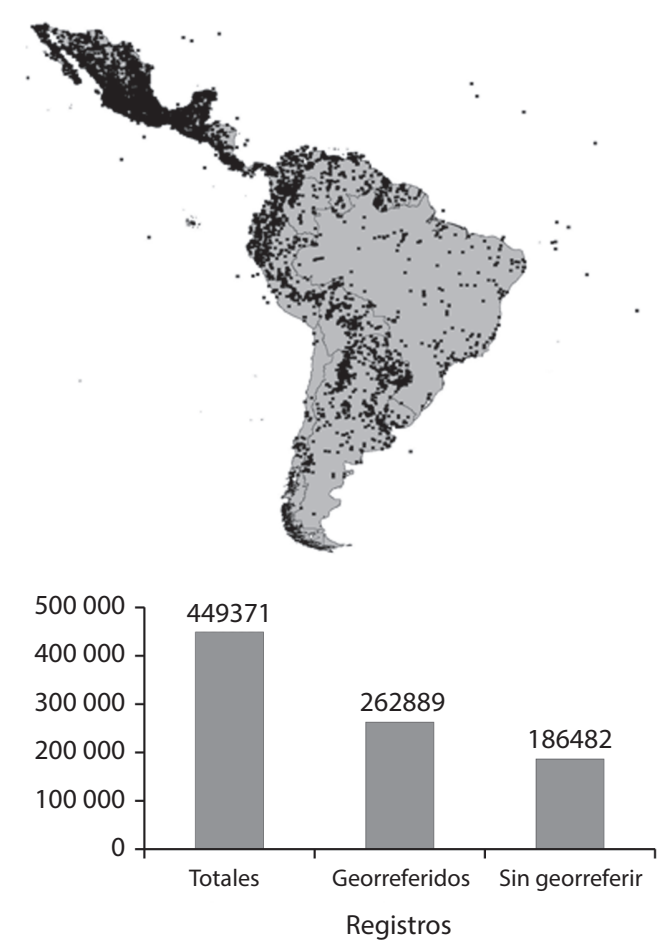

Fig. 1. Distribución espacial de los registros de GBIF georreferidos obtenidos en la consulta de los murciélagos (Chiroptera) en los 21 países de América Latina analizados y número de registros georreferidos y sin georreferir.

Fig. 1. Spatial distribution of the total GBIF bats records from 21 Latin American countries analyzed and number of records within georeference and without georeference.

de cinco países (Brasil, Costa Rica, Ecuador, Guyana y Venezuela) presentaron entre 202 y 260 nombres de especies; mientras que ocho países (Argentina, Belice, Bolivia, Guatemala, Nicaragua, Panamá, Paraguay, Suriname) presentaron entre 102 y 180 nombres; solo El Salvador y Honduras presentaron 98 y 99 nombres de especies respectivamente (Cuadro 1). Los países con los valores más bajos de nombres fueron Guyana Francesa (41), Chile (19) y Uruguay (18) (Fig. 2B).

Evaluación del nombre: Por inspección y comparación de las listas de GBIF y de referencia, se detectaron cinco categorías que indicaron problemas en la correspondencia de los nombres: (1) nombre con escritura incorrecta, por ejemplo para Carollia perspicillata se encontraron registros con nombre Carollia perpicillata o para Antrozous pallidus registros con nombre Antrozous palliduds; (2) sinónimos o nombres sin actualizar como en el caso de Artibeus fuliginosus, considerado una sinonimia de Artibeus obscurus (Haynes \& Lee 2004, Gardner 2008); (3) especie con distribución fuera del continente por ejemplo los registros de Miniopterus schreibersii sin distribución en América; (4) fósiles como Desmodus draculae y (5) ejemplar identificado taxonómicamente hasta familia o género como Sturnira sp. o Sturnira specie. Los registros definidos por las cinco categorías fueron excluidos; los de la categoría 1, considerando que al momento de realizar una consulta por especie estos registros son omitidos. Se eliminaron los de la categoría 2, ya que para la actualización del nombre científico de cualquier tipo de ejemplar es necesaria la revisión directa de los ejemplares o una revisión detallada de cada especie. Los registros con categoría 3 y 4 se excluyeron por no ser parte del objetivo de este estudio. Finalmente, aquellos registros denominados con categoría 5, fueron descartados al ser considerados ambiguos. Con la evaluación de nombres se obtuvo una lista de 313 especies y 243006 registros con nombres coincidentes entre las dos listas (lista GBIF y referencia) para los 21 países. Los registros obtenidos representaron el $54 \%$ del total de registros consultados en GBIF (449 371 reg.) y el $92 \%$ de los registros con georreferencia (262 889 reg.). Los países con el mayor número de especies disponibles fueron: Colombia (173 especies - 14223 reg.), Perú (161 especies - 13217 reg.), México (155 especies - 110199 reg.) y Venezuela (148 especies - 37730 reg.), mientras Guyana Francesa (32 especies - 219 reg.), Chile (9 especies - 356 reg.) y Uruguay (13 especies - 138 reg.) tuvieron el menor número (Figs. 3A y B).

Evaluación geográfica: Se eliminaron 26 793 registros en esta etapa y se conservaron 236096 registros de 304 especies. La mayor reducción de especies fue para Brasil (cinco especies) considerando que después de la 

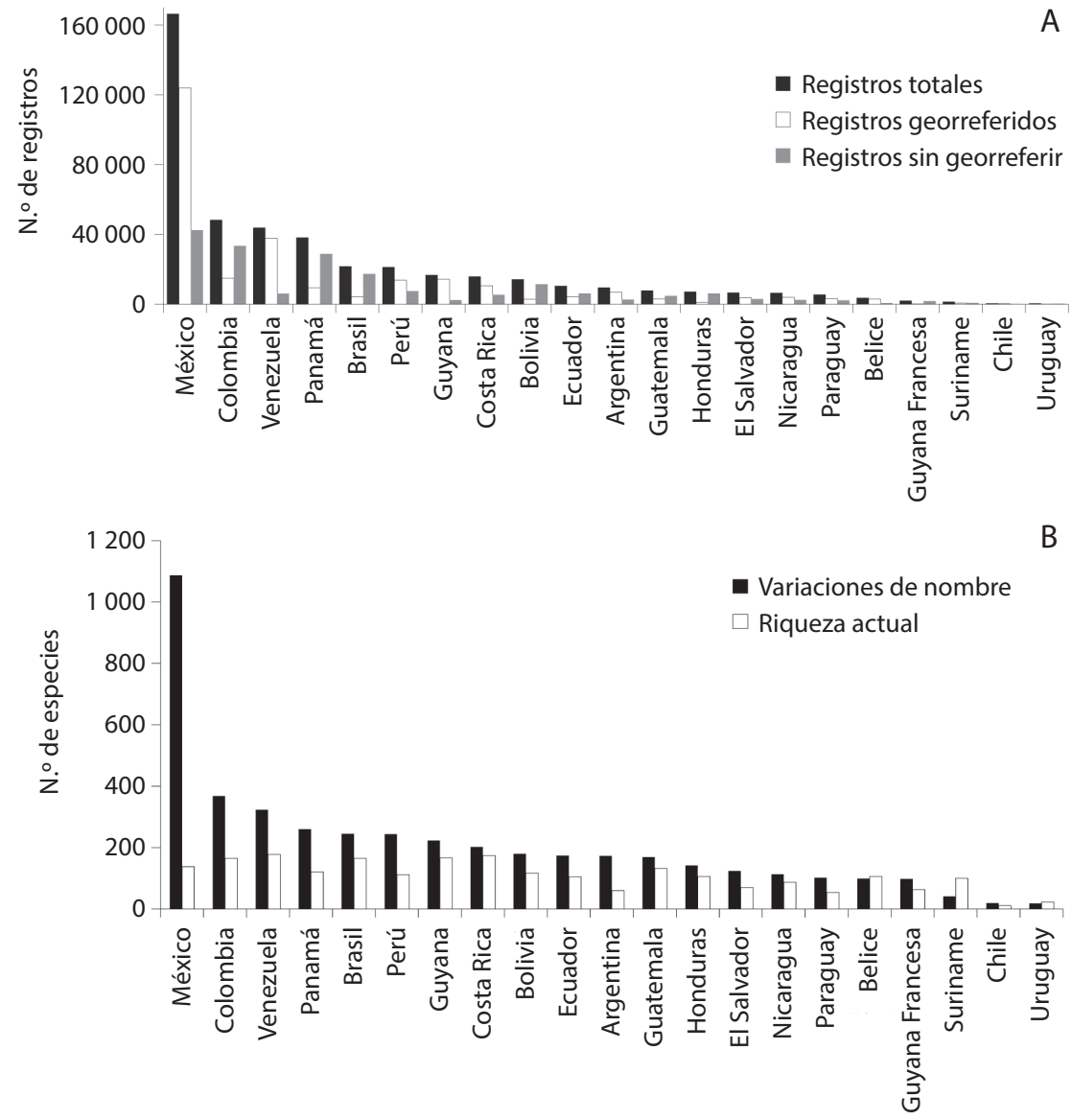

Fig. 2. Número de registros de murciélagos (Chiroptera) para cada país obtenidos durante la consulta en GBIF. (A) Relación de registros totales, georreferidos y sin georreferir. (B) Número de variaciones de nombres científicos y riqueza actual por país.

Fig. 2. Number of the bats (Chiroptera) records to each Latin American countries downloaded from GBIF. (A) Relation of total records, with georeference and without georeference records. (B) Number of scientific names variations and richness for each country.

evaluación de nombres presentaba 114 y luego de la evaluación geográfica tuvo 109 especies (Cuadro 1, Fig. 3B). Países como Bolivia, Guatemala, Guyana, Guyana Francesa, Paraguay, Suriname, Uruguay y Venezuela no sufrieron reducciones de especies en este paso. Los valores finales obtenidos representaron el $52 \%$ del total de registros descargados de GBIF (449 371) (Cuadro 1). El país con el mayor número de especies fue Colombia y con el menor número fue Chile (Cuadro 1). Como se muestra en la Fig. 3B, el mayor número de registros finales los presentó México (109.155 reg.), mientras que Uruguay presentó el menor (130 reg.). Los valores de especies obtenidos después de las dos evaluaciones distan de la riqueza reportada para los 21 países analizados (Cuadro 2). Para Argentina, México y Costa Rica el número de especies obtenidas sobrepasó la riqueza conocida, para los 18 países restantes la riqueza conocida sobrepasó el número de especies con registros validados. Las diferencias entre la riqueza conocida y el número de especies con registros validados de los países se presentaron 


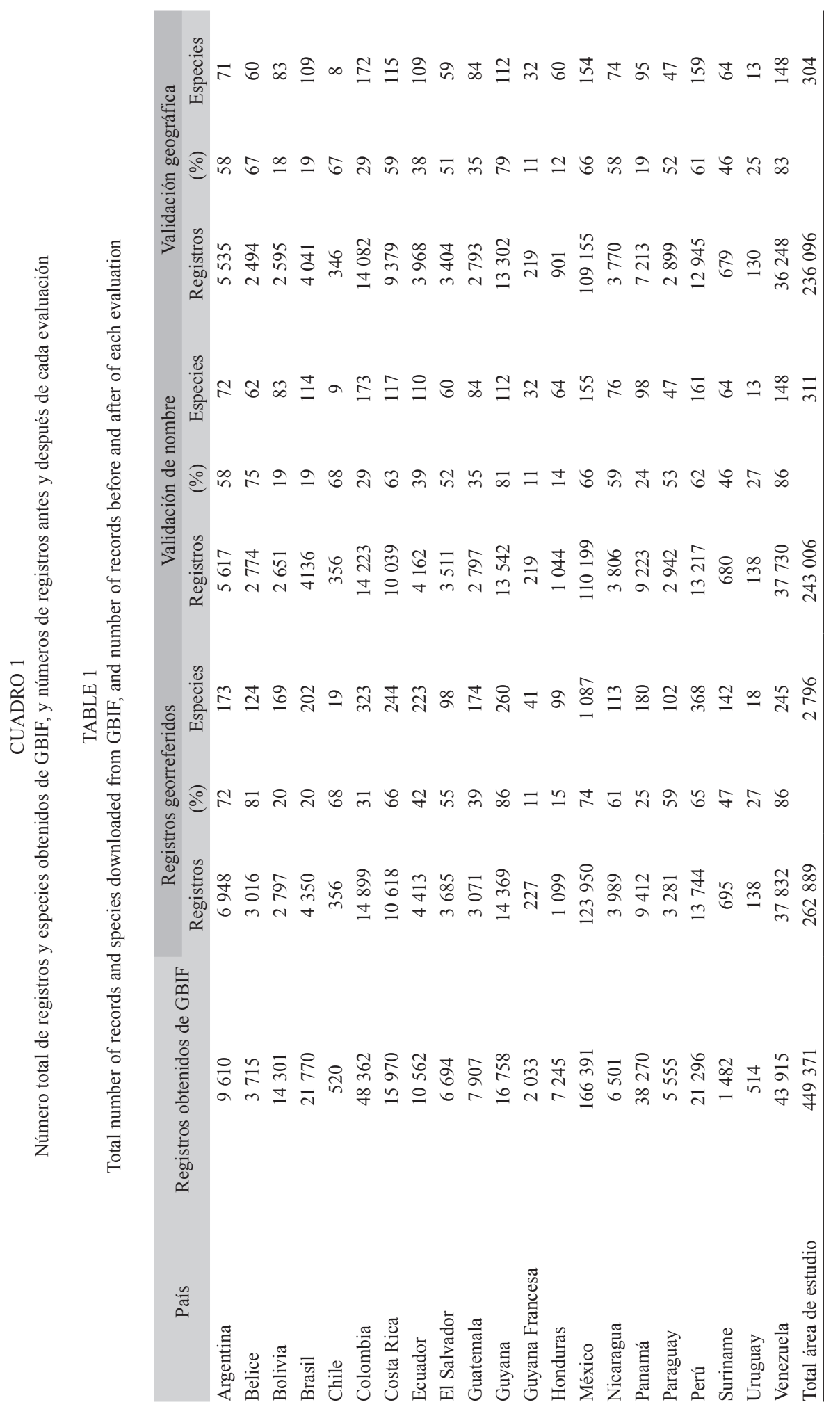



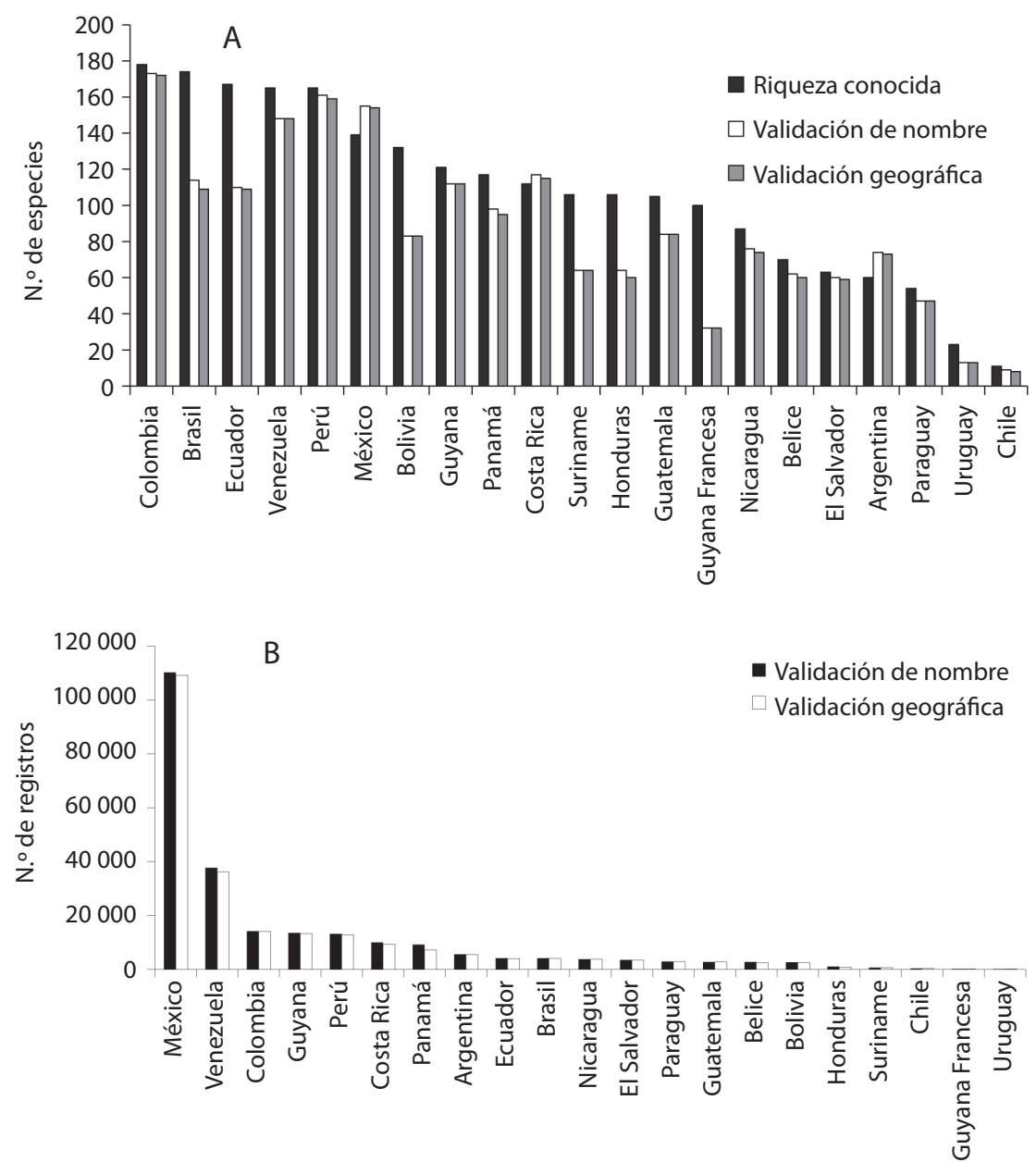

Fig. 3. Número de especies (A) y registros (B) de los murciélagos de los 21 países latinoamericanos luego de la evaluación de nombres científicos.

Fig. 3. Number of species (A) and records (B) of the bats from 21 Latin American countries obtained after nomenclatural evaluation.

en un intervalo de 4 especies (Perú) hasta 68 especies (Guyana Francesa).

\section{Análisis espacial de registros y especies:} Se obtuvieron en total 1756 celdas con registros para los 21 países latinoamericanos. En América del Sur, áreas amplias de la Amazonia Colombiana, el sur de Venezuela, el sur de Argentina, casi todo Brasil y Chile no presentaron registros validados (955 celdas; 54\%). El rango de 1 a 8 especies, integrado por 409 celdas $(23 \%)$ se presentó principalmente en
Argentina - Bolivia y Paraguay; mientras que el rango de 9 a 19 especies suma 171 celdas (9.7\%), con mayor número en México (Fig. 4).

Pocas celdas tienen rangos altos en el número de especies y registros. El 4.4\% (79 celdas) están en el rango de entre 20 y 29 especies, concentrados en Colombia y Ecuador. Se encontraron 30 a 38 especies para el 3\% del área (53 celdas) en países como Guatemala - Ecuador - México y Colombia. El 3.8\% (69 celdas) de celdas presentan el rango 39 a 58 especies, la mayoría de celdas están en 
CUADRO 2

Número de mapas disponibles en IUCN (2010) y valores de riqueza para los murciélagos (Chiroptera) en 21 países de América Latina

TABLE 2

Number of available bats maps on IUCN (2010) and richness of bats in 21 Latin American countries

\begin{tabular}{|c|c|c|c|c|c|}
\hline País & $\begin{array}{c}\text { Mapas de } \\
\text { murciélagos } \\
\text { (IUCN 2010) }\end{array}$ & $\begin{array}{c}\text { \# especies } \\
\text { (Hutson et al. } \\
\text { 2001) }\end{array}$ & $\begin{array}{l}\text { \# especies } \\
\text { obtenidas en } \\
\text { este estudio }\end{array}$ & & Riqueza de murciélagos \\
\hline Argentina & 63 & 57 & 71 & 60 & Barquez, Díaz \& Ojeda, 2006 \\
\hline Belice & 78 & 69 & 60 & 70 & Fenton et al., 2001 \\
\hline Bolivia & 131 & 107 & 83 & 132 & Aguirre et al., 2010 \\
\hline Brasil & 174 & 137 & 109 & 174 & Paglia et al., 2012 \\
\hline Chile & 14 & 10 & 8 & 11 & Palma, 2007 \\
\hline Colombia & 187 & 170 & 172 & 198 & Solari et al., en imprenta. \\
\hline Costa Rica & 106 & 103 & 115 & 112 & Rodríguez-Herrera \& Chavarría, 2010 \\
\hline Ecuador & 152 & 105 & 109 & 167 & Tirira, 2012 \\
\hline El Salvador & 67 & 58 & 59 & 63 & Gallo, 2005 \\
\hline Guatemala & 99 & 94 & 84 & 105 & Pérez, S. 2012. com. pers. \\
\hline Guyana & 132 & 107 & 112 & 121 & Lim et al., 2005 \\
\hline Guyana Francesa & 111 & 102 & 32 & 100 & Lim et al., 2005 \\
\hline Honduras & 100 & 98 & 60 & 106 & Portillo-Reyes, 2007 \\
\hline México & 136 & 137 & 154 & 138 & Ceballos, Arroyo-Cabrales \& Medellín, 2002 \\
\hline Nicaragua & 101 & 88 & 74 & 98 & Medina-Fitoria et al., 2010 \\
\hline Panamá & 124 & 111 & 95 & 117 & Samudio Jr. R., 2012. com. pers. \\
\hline Paraguay & 61 & 49 & 47 & 54 & López-González, 2004 \\
\hline Perú & 163 & 152 & 159 & 165 & Pacheco et al., 2009 \\
\hline Suriname & 119 & 61 & 64 & 106 & Lim, Engstrom \& Ochoa, 2005 \\
\hline Uruguay & 24 & 15 & 13 & 23 & Botto, González \& Rodales, 2008 \\
\hline Venezuela & 163 & 154 & 148 & 165 & Sánchez \& Lew, 2012 \\
\hline
\end{tabular}

México. Únicamente en 21 celdas $(0.011 \%$ del área total; Fig. 4) se sobrepasan las 59 especies (59 a 85 especies), esas celdas se concentraron en Colombia, Costa Rica, Guyana, México y Venezuela (Fig. 4). El 27\% de celdas (477) representaron el menor intervalo de registros (1- 69 registros). Se encontraron siete celdas con el mayor número de registros (3 253 a 6 959), que apenas representan el $0.39 \%$ del area analizada. Los mayores valores de registros están en México, Venezuela y una celda entre Guyana y Suriname (Fig. 5). El análisis de correlación de Spearman indicó una correlación positiva $(\rho=0.99, \mathrm{p}<0.05)$ entre el número de registros y especies, esto implica que el número de especies depende del número de registros.

\section{DISCUSIÓN}

El portal de GBIF provee libremente 449371 registros de los murciélagos (Chiroptera) con distribución en el Neotrópico, que pueden ser consultados alrededor del mundo. Del total de registros analizados, el 58.5\% están georreferidos, pero se debe considerar que dentro de estos, generalmente otra cantidad de registros pueden presentar referencia geográfica imprecisa (Soberón \& Peterson, 2009), lo cual disminuirá aún más el porcentaje obtenido. La mayoría (66\%) de registros de murciélagos están concentrados en cuatro países (Colombia, México, Panamá y Venezuela). México tiene el mayor porcentaje de registros $(37 \%)$, esto sin ser el país con la mayor 

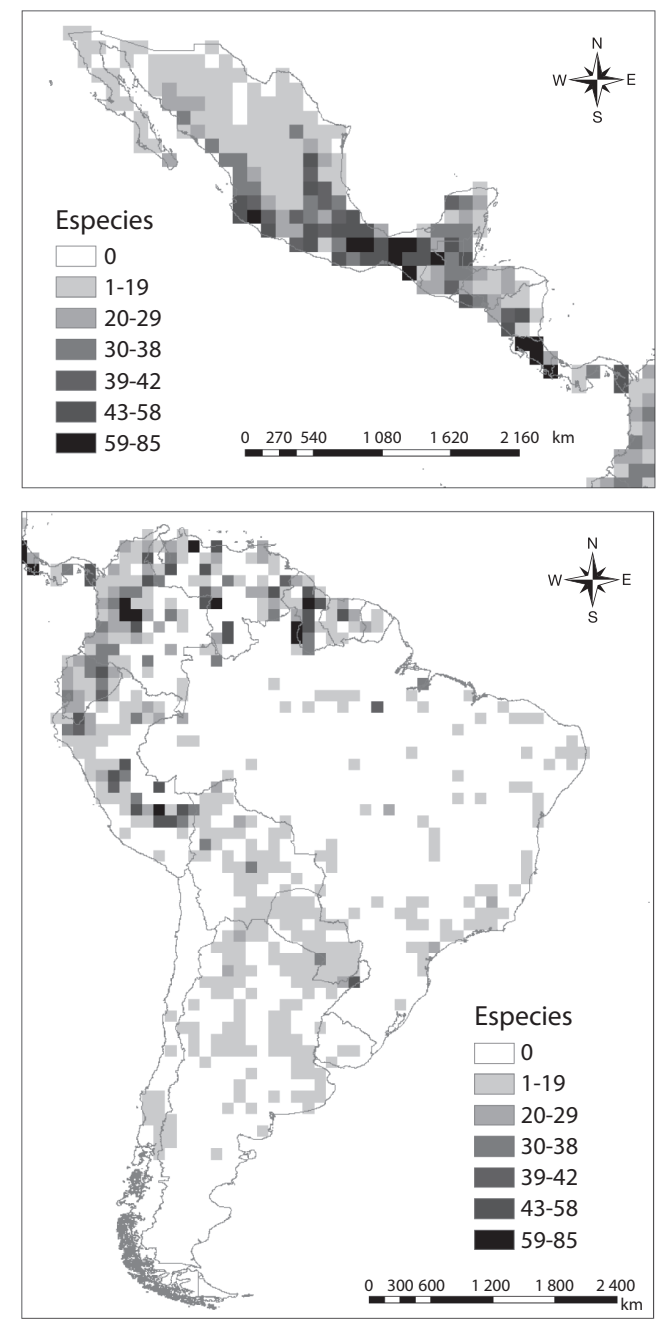

Fig. 4. Número de especies de murciélagos obtenido después de la evaluación de nombre y geográfica en 21 países latinoamericanos, representado en celdas de $1^{\circ}$ latitud $\times 1^{\circ}$ longitud.

Fig. 4. Number of bats species from 21 Latin American countries obtained after nomeclatural and geographical evaluation, represented on $1^{\circ}$ latitude $\times 1^{\circ}$ longitude grid cells.

diversidad de murciélagos, posiblemente los esfuerzos importantes realizados y lo pionero del trabajo de la Comisión Nacional para el Conocimiento y Uso de la Biodiversidad (CONABIO) para concentrar y proveer información de la biodiversidad Mexicana a través
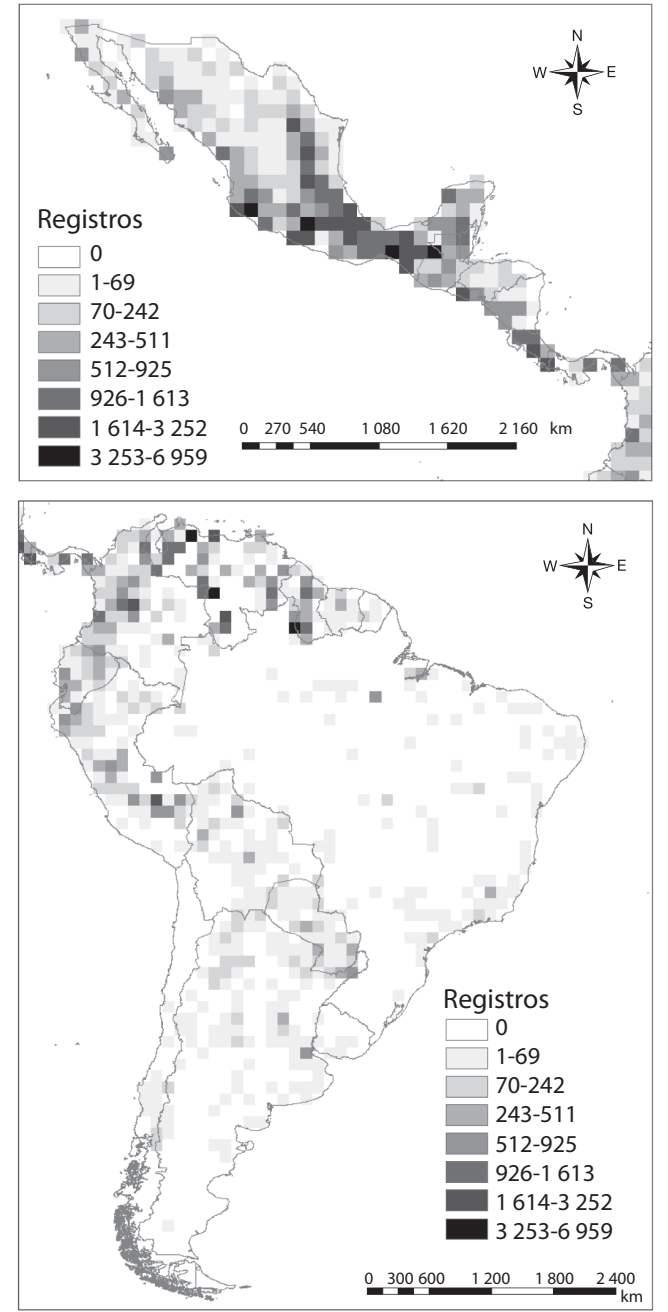

Fig. 5. Número de registros de murciélagos obtenidos después de la evaluación de nombre y geográfica en 21 países latinoamericanos, representada en celdas de $1^{\circ}$ latitud $\times 1^{\circ}$ longitud.

Fig. 5. Number of bats records from 21 Latin American countries obtained after nomeclatural and geographical evaluation, represented on $1^{\circ}$ latitude $\mathrm{x} 1^{\circ}$ longitude grid cells.

de la REMIB (http://www.conabio.gob.mx/ remib/remib.html) sea la razón de esto.

El número de nombres científicos interpretados obtenido inicialmente de GBIF (2 796 nombres) dista de la riqueza de murciélagos (288 especies) reportada para el neotrópico 
(Hutson, Mickleburgh \& Racey, 2001), aun si se consideran otras especies descubiertas en la pasada década (31 especies; Reeder, Helgen, \& Wilson, 2007). Dentro de las inconsistencias propias de las bases de datos están los errores en los nombres de las especies o la referencia geográfica incorrectamente relacionada (Chapman, 2005; Yesson et al., 2007), lo cual aumentó sustancialmente el número de nombres asociados a los registros analizados. México y Colombia fueron los países con los más altos valores en variaciones de nombres científicos y registros geográficamente erróneos; por lo tanto, es necesaria una revisión más detallada de los registros para los dos países identificando las colecciones y los errores que contienen en sus bases de datos. En la literatura se ha comentado sobre la importancia de la validación nomenclatural de los registros disponibles en base de datos en red, para evitar errores en los análisis de biodiversidad (Chapman, 2005; Yesson et al., 2007; Guralnick \& Hill, 2009). Sin embargo es difícil controlar los errores, ya que la función recae directamente en las instituciones que proveen los datos.

La disminución en los porcentajes de nombres después de la evaluación del nombre científico, evidencia la necesidad de continuar el desarrollo de métodos y sistemas que consoliden, organicen y categoricen la información disponible (Neil, 2007; Chapman, 2005; Guralnick \& Hill, 2009), así como también que permitan controlar la incorporación de información taxonómicamente no válida. En América Latina, a nivel local y regional, es necesaria una mayor inversión en los museos de historia natural y colecciones biológicas para disminuir el impedimento taxonómico. Así, los datos disponibles en colecciones de América Latina posiblemente ingresarán con menos sesgos y errores. El uso de información de colecciones biológicas, en algunos casos, se restringe por la ausencia de información asociada a cada ejemplar o por el detalle de la información (Murphey et al., 2004).

En este estudio se consideró que el campo denominado "País interpretado" en los datos descargados de GBIF, representa una correcta relación geográfica de los registros con cada uno de los países. Bajo el anterior supuesto, se obtuvieron 304 nombres de especies válidas que corresponden a 236096 registros, es decir, al finalizar las dos etapas de evaluación, el $52 \%$ del total de registros descargados de GBIF podrían eventualmente ser utilizados para análisis espaciales. Sin embargo, se debe considerar que para una correcta utilización de cualquier dato es necesaria una evaluación más estricta y a menor escala que la validación geográfica aquí realizada, de hecho la calidad de la información condiciona los análisis que se podrían realizar (Meier \& Dikow, 2004; Yesson et al., 2007; Guo et al., 2011).

Las números validados de nombres sobrepasaron la riqueza reportada de países como Argentina, Costa Rica y México; se ha sugerido que errores ocasionados durante la transferencia de los datos de campo a las bases de datos, errores en el datum geográfico e imprecisión en las descripciones de la localidad (Wieczorek, Guo \& Hijmans, 2004) y la actualización de los listados de especies (Escalante et al., 2000; Chapman, 2005) ocasionan malas representaciones de la riqueza de especies en las bases de datos. Para los 18 países restantes la riqueza conocida de especies de murciélagos sobrepasó a la obtenida con la validación de registros de GBIF.

Los países de México y Colombia conservaron el mayor número de especies y registros luego de las dos evaluaciones, mientras que Chile y Uruguay tienen los menores números, sin embargo esos valores tampoco reflejan las riquezas de murciélagos en los cuatro países, aunque estos representen los extremos conocidos de diversidad en el neotrópico (Hutson, Mickleburgh \& Racey, 2001). El país que redujo el mayor número de especies de murciélagos (cinco) con la evaluación geográfica fue Brasil, donde se han reportado problemas similares con plantas, causados por deficiencias en los datos de referencia geográfica como la asignación de coordenadas, el sistema de proyección de los datos, ausencia en los metadatos de los registros, entre otros (Barros et al., 2012). La disminución en el número de especies indicaría 
que hay errores precisión y/o transcripción en el campo "País interpretado" para algunos registros y especies, o que hubo errores entre los sistemas de proyección geográfica del polígono utilizado para la evaluación y los sistemas de georreferencia de los registros (Wieczorek et al., 2004).

Los cambios taxonómicos y las descripciones de nuevas especies ocurren continuamente, por lo tanto tener un número definitivo actual de la riqueza es complejo y sería necesaria la consulta de otras fuentes de información dentro de una revisión más extensa. Los registros geográficos de las 304 especies obtenidas en este estudio, no reflejan la riqueza de murciélagos existente en el Neotrópico, casos similares ocurren con archivos SIG en forma de puntos inicialmente disponibles a través de InfoNatura de NatureServe (Patterson et al., 2007) o los más de 341 mapas poligonales de especies de murciélagos disponibles en la IUCN (2010). En la mayoría de países la riqueza conocida está poco representada en GBIF, como en el caso extremo de Guyana Francesa (100 especies; Lim, Engstrom \& Ochoa, 2005), para el cual se obtuvieron registros válidos tan solo de 32 especies. Otros países como Uruguay (23 especies; Botto, González \& Rodales, 2008) y chile (11 especies; Palma, 2007) que tiene riqueza baja de murciélagos, la información también fue escasa, por efecto de la poca representatividad de la información en la fuente de consulta.

Al realizar el análisis por celdas y no por países, desde el centro de México hasta Panamá, el Chocó y el norte de la zona Andina se encontraron celdas con valores intermedios de registros y especies; lo cual no se acerca a la riqueza de especies de cada país, sino se relaciona con la disponibilidad de registros analizados luego de las evaluaciones. Por otra parte, el 54\% de las celdas analizadas presentan vacíos de información, principalmente en territorios con alta diversidad de especies de murciélagos como la Amazonía Colombiana, el sur de Venezuela y casi todo Brasil (Hutson et al., 2001; Villalobos \& Arita, 2010), o en zonas con menor diversidad por sus condiciones climáticas extremas como la Puna de Chile y la Patagonia Central en Argentina. Tan solo el $0.011 \%$ de las celdas presentaron altos valores de especies (59 a 85 especies), las cuales se concentraron en América Central, concordando con uno de los sitios de alta riqueza de especies de murciélagos identificado por Hutson et al. (2001).

La correlación directa y positiva entre el número de registros y especies explica que la sistematización correcta de información sobre biodiversidad, permitirá en algún momento reflejar la diversidad de murciélagos; con una mayor cantidad de registros de buena calidad y heterogéneamente distribuidos en el espacio geográfico podrían disminuir los vacíos de información encontrados. Los registros de murciélagos para el Neotrópico en GBIF aún distan de representar la diversidad y cobertura geográfica del grupo. Por lo tanto, para considerar los registros de GBIF, en posibles análisis espaciales de diversidad, como es el caso de la implementación de AICOMs, sería necesaria una más detallada y precisa curación de los datos y mayor cooperación de los países latinoamericanos para la liberación de información sobre biodiversidad. En el caso preciso de América del Sur, los vacíos de información pueden ser disminuidos con el incremento en el número de fuentes e instituciones que digitalicen y publiquen datos desde América Latina, esto permitirá avanzar en el procesamiento y análisis de información para el entendimiento de los patrones espaciales de la diversidad de murciélagos.

Es posible que esfuerzos mayores, tanto regionales como locales en América del Sur, dirigidos al desarrollo de las capacidades humanas en áreas como sistemática, taxonomía y análisis espacial, junto al fortalecimiento científico y técnico de las colecciones biológicas, permitan el desarrollo de sistemas para la evaluación y validación de la información. La eficiencia en el uso de la información libre sobre biodiversidad depende de la calidad de los datos, por lo tanto, proveedores y usuarios deben unir esfuerzos para lograr una alta consistencia taxonómica y geográfica antes de suministrar o analizar la información; pero finalmente es responsabilidad de aquellos que 
utilizan la información desarrollar protocolos para su validación y verificación.

\section{AGRADECIMIENTOS}

A Rafael Samudio Jr., Sergio Pérez, Ignacio Ferro y Héctor Ramírez-Chaves por la información suministrada sobre los murciélagos de Panamá, Guatemala, Argentina y Colombia, respectivamente. A Juan José Morrone, Víctor Sánchez Cordero y a tres revisores anónimos por sus comentarios y sugerencias para mejorar este documento. EANU agradece al posgrado en Ciencias de la Universidad Nacional Autónoma de México (UNAM) y al programa de becas 2011-2013 del Consejo Nacional de Ciencia y Tecnología de México (CONACyT), por el soporte dado para el desarrollo de la investigación y escritura de este documento.

\section{RESUMEN}

Los esfuerzos globales para digitalizar los datos de ocurrencia de la biodiversidad en colecciones, museos y otras instituciones han estimulado el desarrollo de herramientas para mejorar el conocimiento y conservación de la biodiversidad. La "Global Biodiversity Information Facility" GBIF permite el acceso a más de 321 millones de registros, alojados en 379 instituciones. Los murciélagos neotropicales son un grupo muy diverso y especializado y la información geográfica del grupo se ha incrementado desde hace unos años, pero son pocos los reportes acerca del tema. El objetivo de este estudio fue analizar el número de registros disponibles en GBIF de los murciélagos neotropicales de 21 países de América. Por lo tanto, se evaluó la consistencia del nombre científico y la calidad geográfica a escala de país. Además, se evaluaron vacíos de información sobre una grilla de $1^{\circ}$ latitud y $1^{\circ}$ longitud. Hubo cerca de 1/2 millón de registros, de los cuales el 58\% no incluyeron coordenadas geográficas; el 52\% pasaron las dos evaluaciones. Se estimó que el 54\% del área geográfica analizada no tiene registros; los vacíos están en centros de biodiversidad como la Amazonía y la Patagonia. En conclusión nuestros resultados sugieren que los datos disponibles en GBIF tienen sesgos geográficos y en los nombres científicos. Los datos de GBIF representan parcialmente las riquezas de murciélagos, además los principales vacíos de información se encuentran en América del Sur.

Palabras clave: registros, base de datos, número de especies, riqueza, murciélagos, América Latina.

\section{REFERENCIAS}

Aguirre, L. F., Mamani, C. J., Barbosa-Márquez, K., \& Mantilla-Meluk, H. (2010). Lista actualizada de murciélagos de Bolivia. Revista Boliviana de Ecología y Conservación Ambiental, 27, 1-7.

Barquez, R. M., Díaz, M. M., \& Ojeda, R. A. (Eds.). (2006). Mamiferos de Argentina: sistemática y distribución. Tucumán: Sociedad Argentina para el Estudio de los Mamíferos.

Barros, F. S., Fernandes, R. A., Moraes, M. A., Pougy, N. M., Caram, J. S., Dalcin, E. C., \& Martinelli, G. (2012). Spatial data quality of herbarium datasets and implications for decision-making on biodiversity conservation in Brazil. Proceeding of the 10th International Symposium on Spatial Accuracy Assessment in Natural Resources and Environmental Science, 209-214.

Boitani, L., Maiorano, L., Baisero, D., Falcucci, A., Visconti, P., \& Rondinini, C. (2012). What spatial data do we need to develop global mammal conservation strategies? Philosophical Transactions of the Royal Society B: Biological Sciences, 366, 2623-2632.

Botto, G., González, E. M., \& Rodales, A. L. (2008). Promops centralis Thomas, 1915, nuevo género y especie de murciélago para Uruguay (Mammalia, Molossidae). IX Jornadas de Zoología del Uruguay.

Ceballos, G., Arroyo-Cabrales, J., \& Medellín, R. A. (2002). The mammals of México: Composition, distribution and conservation status. Occasional papers, Texas Tech University, 218, 1-27.

Chapman, A. D. (2005). Principles and methods of data cleaning. Report for the Global Biodiversity Information Facility 2004. Copenhagen: GBIF.

Costello, M. J., Michener, W. K., Gahegan, M., Zhi-Qiang, Z., Phil, B., \& Chavan, V. (2012). Quality assurance and intellectual property rights in advancing biodiversity data publications ver. 1.0, Global Biodiversity Information Facility, Copenhagen. Secretaria GBIF.

Crisp, M. D., Laffan, S., Linder, H. P., \& Monro, A. (2001). Endemism in the Australian flora. Journal of Biogeography, 28, 183-198.

de Diego, J. 2010. ddTraDa Básico (ver.1.54). [Software de cómputo]. Chile. Recuperado el 20 de agosto de 2011, de www.ddTraDa.cl.

Edwards, J. (2004). Research and societal benefits of the Global Biodiversity Information Facility. BioScience, 54(6), 486-487.

Escalante, T. (2005). Las bases de datos curatoriales y el estudio espacial de la biodiversidad: Un ejemplo con los mamíferos terrestres de México. In J. Llorente, J.J. Morrone (Eds.), Regionalización biogeográfica en Iberoamérica y tópicos afines: Primeras Jornadas Biogeográficas de la Red Iberoamericana de 
Biogeografia y Entomología Sistemática (339-350). México: CYTED-UNAM-Conabio.

Escalante, T., D., Espinosa, \& Morrone, J. J. (2002). Patrones de distribución geográfica de los mamíferos terrestres de México. Acta Zoológica Mexicana (n. s.), 8, 47-65.

Escalante, T., Llorente, J., Espinosa, D., \& Soberón, J. (2000). Bases de datos y sistemas de información: aplicaciones en biogeografía. Revista de la Academia Colombiana de Ciencias Exactas, Físicas y Naturales, 24(92), 325-341.

ESRI. 2008. ArcGis (v 9.3). [Software de cómputo]. Environmental Systems Research Institute: Redlands. California.

Fenton, M. B., Bernard, E., Bouchard, S., Hollis, L., Johnston, D. S., Lausen, C. L., Ratcliffe, J. M., Riskin, D. K., Taylor, J. R., \& Zigouris, J. (2001). The bat fauna of Lamanai, Belice: roosts and trophic roles. Journal of Tropical Ecology, 17, 511-524.

GADM 2.0. 2011. (13 de abril de 2012). Base de datos mundial sobre áreas administrativas.

Gallo, M. (2005). Estado del conocimiento de la Biodiversidad en El Salvador. Documento final. Ministerios de Medios Ambiente y Recursos Naturales. Instituto Nacional de Biodiversidad (INBio). El Savador.

Gardner, A. L. (Ed.). (2008). Mammals of South America: Marsupials, xenarthrans, shrews and bats. The University of Chicago Press, Chicago.

Gaubert, P., Papes, M., \& Peterson, A. T. (2006). Natural history collections and the conservation of poorly known taxa: Ecological niche modeling in central African rainforest genets (Genetta spp). Biological Conservation, 130, 106-117.

Guo, Q., Lia, W., Liu, Y., \& Tong, D. (2011). Predicting potential distributions of geographic events using one-class data: concepts and methods. International Journal of Geographical Information Science, 25(10), 1697-1715.

Guralnick, R., \& Hill, A. (2009). Review: Biodiversity informatics-automated approaches for documenting global biodiversity patterns and processes. Bioinformatics, 25(4), 421-428.

Haynes, M. A., \& Lee, T. E. (2004). Artibeus obscurus. Mammalian Species, 752, 1-5.

Hijmans, R. J., \& van Etten, J. (2012). (03 de marzo de 2012) Package 'raster': Geographic analysis and modeling with raster data. [Software de cómputo].

Hijmans, R. J., L. Guarino, C. Bussink, P. Mathur, M. Cruz, I. Barrantes, \& Rojas, E. 2004. DIVA-GIS Versión 4. Sistema de información geográfica para el análisis de distribución de especies. Manual. International Potato Center. Lima, Perú.
Hutson, A. M., Mickleburgh, S. P., \& Racey, P. A. (Eds.). (2001). Microchiropteran bats: global status survey and conservation action plan. IUCN/SSC Chiroptera Specialist Group. IUCN, Gland, Switzerland and Cambridge, UK.

IUCN. 2010. IUCN Red List of Threatened Species. Version 2010.4. Cambridge CB3 0DL, United Kingdom.

Kozak, K. H., Graham, C., \& Wiens, J. J. (2008). Integrating GIS-based environmental data into evolutionary biology. Trends in Ecology and Evolution, 23(3), 1-8.

Lane, M. A. (2007). GBIF Strategic and Operational Plans 2007-2011: From Prototype towards Full Operation. Copenhagen: Global Biodiversity Information Facility.

Lim, B. K., Engstrom, M. D., \& Ochoa, J. G. (2005). Mammals. In T. Hollowell \& R. P. Reynolds (Eds.), Checklist of the Terrestrial Vertebrates of the Guiana Shield (pp. 77-92). USA: Bulletin of the Biological Society of Washington 13.

López-González, C. (2004). Ecological zoogeography of the bats of Paraguay. Journal of Biogeography, 31, 33-45.

Medina-Fitoria, A., Saldaña, O., McCarthy, T. J., \& Vilchez, S. (2010). Nuevos reportes y comentarios históricos de murciélagos (Mammalia: Chiroptera) para la fauna de Nicaragua. Revista biodiversidad y áreas protegidas, 2, 93-101.

Meier, R., \& Dikow, T. (2004). Significance of specimen databases from taxonomic revisions for estimating and mapping the global species diversity of invertebrates and repatriating reliable specimen data. Conservation Biology, 18, 478-488.

Murphey, P. C., Guralnick, R. P., Glaubitz, R., Neufeld, D., \& Ryan, J. A. (2004). Georeferencing of museum collections: a review of problems and automated tools, and the methodology developed by the Mountain and Plains Spatio-Temporal Database-Informatics Initiative (Mapstedi). Phyloinformatics, 1, 1-29.

Neil, I. (2007). Biodiversity informatics: organizing and linking information across the spectrum of life. Briefings in Bioinformatics, 8(5), 347-357.

Pacheco, V., Cadenillas, R., Salas, E., Tello, C., \& Zeballos, H. (2009). Diversidad y endemismo de los mamíferos del Perú. Revista Perúana de Biología, 16(1), 5-32.

Paglia, A. P., Fonseca, G. A. B., da Rylands, A. B., Herrmann, G., Aguiar, L. M. S., Chiarello, A. G., Leite, Y. L. R., Costa, L. P., Siciliano, S., Kierulff, M. C. M., Mendes, S. L., da C. Tavares V., Mittermeier, R. A., \& Patton, J. L. (2012). Annotated Checklist of Brazilian Mammals. Occasional Papers in Conservation Biology 6. Conservation International, Arlington.

Palma, R. E. (2007). Estado actual de la mastozoología en Chile-Editorial. Mastozoología neotropical, 14(1), 5-9. 
Patterson, B. D., Ceballos, G., Sechrest, W., Tognelli, M. F., Brooks, T., Luna, L., Ortega, P., Salazar, I., \& Young, B. E. (2007). Digital Distribution Maps of the Mammals of the Western Hemisphere, version 3.0. NatureServe, Arlington, Virginia, U.S.A.

Petersen, F. T., Meier, R., \& Nykjaer, M. (2003). Testing species richness estimation methods using museum label data on the Danish Asilidae. Biodiversity Conservation, 12, 687-701.

Ponder, W. F., Carter, G. A., Flemons, P., \& Chapman, R. R. (2001). Evaluation of museum collection data for use in biodiversity assessment. Conservation Biolology, 15(3), 648-657.

Portillo-Reyes, H. O. (2007). Recopilación de la información sobre la biodiversidad de Honduras. (Informe final de Consultaría). Tegucigalpa: INBIO-DiBio.

RELCOM. (2010). Estrategia para la conservación de los murciélagos de Latinoamérica y el Caribe.

Reeder, D. M., Helgen, K. M., \& Wilson, D. E. (2007). Global trends and biases in new mammal species discoveries. Occasional Papers, Museum of Texas Tech University 269, 1-35.

Rodríguez-Herrera, B., \& Chavarría, C. R. (2010). Educando para conservar. Boletín Red Latinoamericana para la Conservación de los Murciélagos, 1(2), 1-17.

R. Version 2.14.1. (2011). Development Core Team. R: A language and environment for statistical computing. [Software de cómputo]. R Foundation for Statistical Computing, Vienna, Austria.

Sánchez, H. J., \& Lew, D. (2012 [2010]). Lista actualizada y comentada de los mamíferos de Venezuela. Memoria de la Fundación La Salle de Ciencias Naturales, 173-174: 173-238.

Shaffer, H. B., Fisher, R. N., \& Davidson, C. (1998). The role of natural history collections in documenting species declines. Trends in Ecology and Evolution, 13: 27-30.

Simmons, N. B. (2005). Order Chiroptera. In D. E. Wilson, \& Reeder D. M. (Eds.), Mammals Species of the World: a taxonomic and geographic reference (pp. 2142). Baltimore, Maryland: Johns Hopkins University Press.

Soberón, J., \& Peterson, A. T. (2009). Monitoring biodiversity loss with primary species-occurrence Data: Toward National-level Indicators for the 2010 Target of the Convention on Biological Diversity. AMBIO: A Journal of the Human Environment, 38(1), 29-34.

Sokal, R. R., \& Rolf, F. J. (1995). Biometry. Nueva York: Freeman and Company.

Solari, S., \& Baker, R. J. (2006). Mitochondrial DNA sequence, karyotypic, and morphological variation in the Carollia castanea species complex (Chiroptera: Phyllostomidae) with description of a new species. Occasional Papers, Museum of Texas Tech University, 254(14), 1-16.
Solari, S., Hoofer, S. R., Larsen, P. A., Brown, A., Bull, R. J., Guerrero, J. A., Ortega, J., Carrera, J. P., Bradley, R. D., \& Baker, R. J. (2009). Operational criteria for genetically defined species: analysis of diversification of the small fruit-eating bats, Dermanura (Phyllostomidae: Stenodermatinae). Acta Chiropterologica, 11(2), 279-288.

Tirira, D. G. (2012). Lista actualizada de especies de mamíferos en el Ecuador / Updapted species check list of Mammals in Ecuador ver. 2012.1. Quito: Editorial Murciélago Blanco y Fundación Mamíferos y Conservación.

Velazco, P. M. (2005). Morphological Phylogeny of the bat Genus Platyrrhinus Saussure, 1860 (Chiroptera: Phyllostomidae) with the description of four new species. Fieldiana Zoology (n.s.), 105(1535), 1-53.

Velazco, P. M., \& Gardner, A. L. (2009). A new species of Platyrrhinus (Chiroptera: Phyllostomidae) from western Colombia and Ecuador, with emended diagnoses of $P$. aquilus, $P$. dorsalis, and $P$. umbratus. Proceedings of the Biological Society of Washington, 122(3), 249-281.

Velazco, P. M., Gardner, A. L., \& Patterson, B. D. (2010). Systematics of the Platyrrhinus helleri species complex (Chiroptera: Phyllostomidae), with descriptions of two new species. Zoological Journal of the Linnean Society, 159(3), 785-812.

Velazco, P. V., \& Simmons, N. B. (2011). Systematics and taxonomy of Great Striped-Faced bats of the genus Vampyrodes Thomas, 1900 (Chiroptera: Phyllostomidae). American Musseum Novitates, 3710, 1-35.

Villalobos, F., \& Arita, H. (2010). The diversity field of New World leaf-nosed bats (Phyllostomidae). Global Ecology and Biogeography, 19, 200-211.

Wieczorek, J. R., Guo, Q., \& Hijmans, R. J. (2004). The point-radius method for georeferencing point localities and calculating associated uncertainty. International journal of geographical information science, 18, 745-767.

Wilson, D. E., \& Reeder, D. M. (Eds.). (2005). Mammal Species of the World: A Taxonomic and Geographic Reference. Baltimore: Johns Hopkins University Press.

Yesson, C., Brewer, P. W., Sutton, T., Caithness, N., Pahwa, J. S., Burgess, M., Gray, W. A., White, R. J., Jones, A. C., Bisby, A. F., \& Culham, A. (2007). How global is the global biodiversity information facility? PLoS One, 2(11), e1124.

Zurc, D., \& Velasco, P. M. (2010). Análisis morfológico y morfométrico de Carollia colombiana Cuartas et al. 2001 y C. monohernandezi Muñoz et al. 2004 (Phyllostomidae: Carollinae) en Colombia. Chiroptera Neotropical, 16(1), 567-572. 


\section{ANEXO I}

Lista de instituciones que contribuyen con registros de colecciones de murciélagos usados en este análisis

\section{APPENDIX I}

List of institutions providing bats specimen data used in this analysis

\section{GBIF}

- Mammal specimens, http://data.gbif.org/datasets/resource/181

- Mammals, http://data.gbif.org/datasets/resource/8439

- Registros biológicos en Áreas protegidas obtenidos de documentos impresos, http://data.gbif.org/datasets/ resource/10869

- $\quad$ TCWC Vertebrate Collections, http://data.gbif.org/datasets/resource/8962

- $\quad$ Tissues Specimens, http://data.gbif.org/datasets/resource/9163

- $\quad$ MCZ Mammalogy Collection, http://data.gbif.org/datasets/resource/581

- Collection Mammalia SMF, http://data.gbif.org/datasets/resource/8313

- Colección Nacional de Mastozoología - Museo Argentino de Ciencias Naturales 'Bernardino Rivadavia', http://data. gbif.org/datasets/resource/9115

- $\quad$ NMNH Vertebrate Zoology Mammals Collections, http://data.gbif.org/datasets/resource/1837

- Colección de Mastozoología, http://data.gbif.org/datasets/resource/13547

- Mammal specimens, http://data.gbif.org/datasets/resource/657

- Mammal specimens, http://data.gbif.org/datasets/resource/582

- Vertebrate specimens, http://data.gbif.org/datasets/resource/1795

- Mammal Collection, http://data.gbif.org/datasets/resource/148

- Vertebrate specimens, http://data.gbif.org/datasets/resource/541

- $\quad$ FMNH Mammals Collections, http://data.gbif.org/datasets/resource/177

- Plan de vertebrados de la Patagonia, http://data.gbif.org/datasets/resource/13514

- $\quad$ Estación Biológica Donana - CSIC, Mammal Collection, http://data.gbif.org/datasets/resource/1759

- CNMA/Colección Nacional de mamíferos, http://data.gbif.org/datasets/resource/776

- Mammals (NRM), http://data.gbif.org/datasets/resource/1041

- Zoological specimens, http://data.gbif.org/datasets/resource/9104

- $\quad$ SysTax, http://data.gbif.org/datasets/resource/1875

- $\quad$ Bird and Mammal specimens, http://data.gbif.org/datasets/resource/1846

- Bishop Museum Natural Sciences Data, http://data.gbif.org/datasets/resource/54

- Peabody Mammalogy DiGIR Service, http://data.gbif.org/datasets/resource/1022

- Zoological Museum Amsterdam, University of Amsterdam (NL) Mammalia, http://data.gbif.org/datasets/ resource/12489

- Mammals of the Gothenburg Natural History Museum, http://data.gbif.org/datasets/resource/1047

- $\quad$ MVZ Mammal Catalog, http://data.gbif.org/datasets/resource/8121

- Mammal specimens, http://data.gbif.org/datasets/resource/986

- $\quad$ Paleobiology Database, http://data.gbif.org/datasets/resource/563

- Mammal specimens, http://data.gbif.org/datasets/resource/559

- Mammals Specimens, http://data.gbif.org/datasets/resource/631

- Mammal Collection, http://data.gbif.org/datasets/resource/984

- Mammal Specimens, http://data.gbif.org/datasets/resource/124

- Division of Mammals, Museum of Southwestern Biology, Albuquerque, NM., http://data.gbif.org/datasets/ resource $/ 980$

- Mammal Collection Catalog, http://data.gbif.org/datasets/resource/162

- Avistajes de especies de valor especial en Áreas protegidas del noreste de Argentina, http://data.gbif.org/datasets/ resource/13513

- Mammal specimens, http://data.gbif.org/datasets/resource/801

- Vertebrados de valor especial en áreas protegidas de La Argentina, http://data.gbif.org/datasets/resource/10868

- Mammal collection, Natural History Museum, University of Oslo, http://data.gbif.org/datasets/resource/8067 
